\title{
A Visible Light-triggered Ferroptosis Probe: Visualization, Endoplasmic Reticulum Localization and Photo-induced Lipid
}

\section{Peroxidation}

Zhiming Xing ${ }^{1 \dagger}$, Jiangyu Yan ${ }^{2 \dagger}$, Yongxiang Miao ${ }^{1}$, Yawen Ruan ${ }^{2}$, Youkang Zhou ${ }^{1}$, Yingqun Tang ${ }^{1}$, Guorui Li $^{2}$, Jing Huang $^{2^{*}}$, Yiyuan Peng ${ }^{1 *}$, and Zhibin Song ${ }^{{ }^{*}}$

${ }^{1}$ Key Laboratory for Green Chemistry of Jiangxi Province, Key Laboratory of Functional Small Molecules for Ministry of Education, Jiangxi Normal University, Nanchang 330022, China.

2 Institute of Chemical Biology and Nanomedicine (ICBN), State Key Laboratory of Chemo/Biosensing and Chemometrics, College of Biology, Hunan University, Changsha 410082, China

$\dagger$ These authors contributed equally to this work.

*Corresponding Authors: Email Address: Jing Huang: huangjing16@hnu.edu.cn; Yiyuan Peng: yypeng@jxnu.edu.cn; Zhibin Song: zbsong@jxnu.edu.cn

\begin{abstract}
:
Accumulation of lethal lipid peroxidation plays the central role in ferroptosis initiation and execution. However, it remains unclear whether the location of lipid peroxidation accumulation in subcellular organelles contributes to ferroptosis initiation due to the lack of specific small-molecule probe. Here we report a quinazolinone-pyridine difluoroboron complex (BODIQPys) with vinyltriphenylamine modification, BODIQPy-TPA, as a near-infrared (NIR) bioimaging and photo-induced ferroptosis bifunctional probe. BODIQPy-TPA with D- $\pi$-A structure exhibits a good endoplasmic reticulum (ER) localization and photo-activated lipid reactive oxygen species (ROS) generation. The photo-induced ferroptosis is confirmed by morphological changes of live cell fluorescence imaging, suppression of cell death by a ferroptosis inhibitor (Fer-1) and down-regulation of ferritin heavy polypeptide 1 (FTH1). This study is the first example of visual photo-induced ferroptosis by small organic molecular probe, which provides a promising tool for further investigation of the direct relationship between lipid peroxidation accumulation and ferroptosis. Moreover, ferroptosis induced by photodynamic therapy (PDT) process in ER will be a good strategy to improve the efficiency of PDT for apoptosis-resistant tumor cells.
\end{abstract}

\section{INTRODUCTION}

Ferroptosis is a recently recognized form of programmed cell death that is different from other forms of cell death in morphological, genetic and biochemical levels[1-4]. Caused by iron-dependent accumulation of lethal lipid peroxidation, ferroptosis can generate mild immunogenicity and cannot be suppressed by drug resistance related mutations in tumor cells $[5,6]$. Thus, the development of ferroptosis inducers becomes a promising strategy for cancer treatment, especially to drug-resistant tumors[7-9]. More than that, ferroptosis inducers as chemical probes also provide effective tools to explore and understand the mechanism of ferroptosis initiation and execution[4]. For instance, the glutathione peroxidase 4 (GPX4) inhibition, GSH depletion and cystine starvation (the GPX4-GSH-cysteine axis) have been identified as an important pathway for inducing ferroptosis by small-molecule reagents such as erastin and RSL3[1, 10, 11]. Depleting lipophilic antioxidant ubiquinol/coenzyme $\mathrm{Q}_{10}\left(\mathrm{CoQ}_{10}\right)$ has been reported as another pathway for 
inducing ferroptosis by compounds FIN56 and iFSP1[12,13]. On the other side, ferroptosis is also achieved by affecting iron metabolism[14]. Excess redox-active iron in the process of iron metabolism is able to catalyze the Fenton reaction to accelerate the lipid reactive oxygen species (ROS) production and lead to ferroptosis[1,8]. Accordingly, some iron containing nanomaterials as ferroptosis inducers were then developed for ferroptosis-enhanced tumor therapy[15-17]. Therefore, the development of new types of ferroptosis inducers not only provides a powerful tool for exploring the mechanism of ferroptosis, but also provides benefits to the treatment of tumors.

Despite the excess of lipid-based ROS that has been proved to be the central role for initiation of ferroptosis[18], it is still unclear whether the enrichment of lipid peroxide in specific organelles leads to cell death[3,4,6]. Mitochondria, as the very important organelle in lipid metabolism and cellular ROS generation, have been proved to be a crucial player in cysteine deprivation-induced ferroptosis[19]. Nevertheless, there are some results which indicate that the role of mitochondria in ferroptosis caused by other forms of cellular ROS aggregation and GPX4 inhibition is not significant $[4,20]$. In addition, ferrostatins, as ferroptosis inhibitors, can still inhibit ferroptosis in mitochondria deficient cells[20]. These results indicated that mitochondria may not be the primary site of lipid peroxidation for ferroptosis initiation except for cysteine deprivation pathway. Endoplasmic reticulum, as a source of lipids for most membranes in other organelles, is another important organelle in the lipid metabolism regulation. Recently, NADPH-cytochrome P450 reductase (POR) in ER has been reported to play a substantial role in membrane phospholipids peroxidation and ferroptosis execution[21,22]. In addition, it is worth noting that ferrostatins located in the ER may be the key factor for prevention of lipid peroxidation and ferroptosis suppression[23]. Therefore, the development of novel chemical probes with good ROS generation ability and ER localization would help to further understand the ferroptosis initiation and execution.

Photo-triggered ROS production by photosensitizers such as organic dyes, metal complexes and nanoparticles is a very efficient way for photodynamic therapy (PDT) and chemical transformation[24-27]. However, most of the reported photosensitizers caused apoptosis rather than ferroptosis through light-induced ROS production, which led to the fact that tumor cells could enhance the resistance to this common cell death mode of PDT by upregulating the expression of antioxidants[28,29]. Although some photosensitizers as PDT agents have been developed to enhance tumor therapy by the synergism of ferroptosis and apoptosis recently[29], those PDT agents are not suitable as chemical probe for investigation of the process of ferroptosis initiation due to the complicated roles of ROS produced by such photosensitizers in cell death. To the best of our knowledge, the small organic molecule PDT agents that located in endoplasmic reticulum as ferroptosis inducers were rare[30]. In this work, we designed a novel quinazolinone-based difluoroboron complex (BODIQPy-TPA). This probe showed good ability of ER localization and photoinduced ROS generation, which could effectively realize the photo-activated ferroptosis in B16 cells. In addition, BODIQPy-TPA showed good near infrared (NIR) fluorescence emission in live cells, which can be used as a NIR bioimaging probe to observe the process of ferroptosis.

\section{RESULTS AND DISCUSSION}

\section{Synthesis and optical properties of BODIQPy-TPA}

Based on our previous results[31,32], quinazolinone-pyridine difluoroboron complex (BODIQPys) 
exhibited more electron deficiency compared with boron dipyrromethene (BODIPY), which can be a better electron acceptor. Therefore, we aim to construct a D- $\pi$-A structure of BODIQPys with vinyltriphenylamine group modification (Figure 1). The introduction of triphenylamine (TPA) group can improve the interaction between BODIQPys and lipids $[33,34]$.
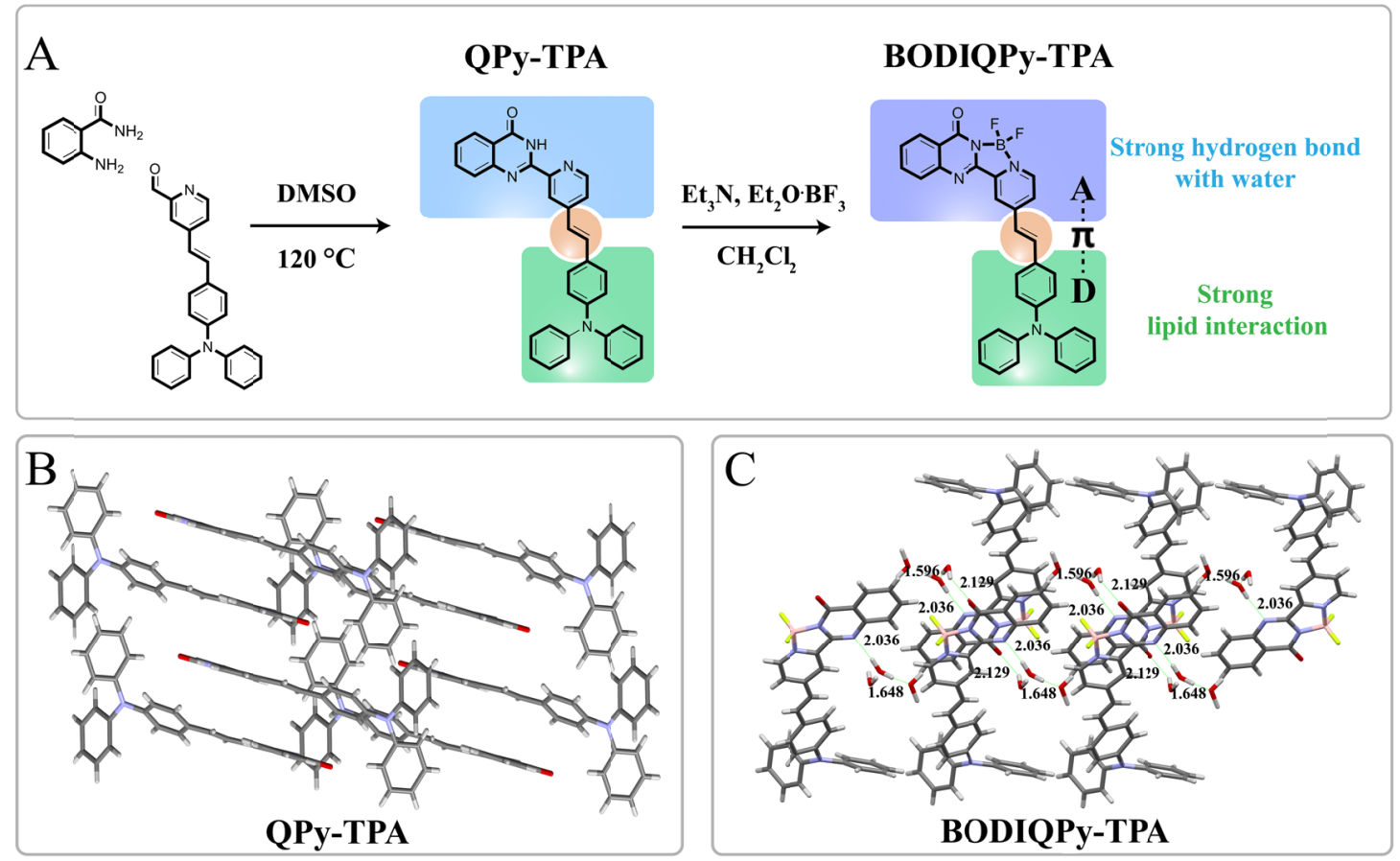

Figure 1. Synthesis and single crystal structure of QPy-TPA and BODIQPy-TPA

BODIQPy-TPA was prepared via two simple steps and characterized by ${ }^{1}$ HNMR and HRMS. The single crystal of BODIQPy-TPA (CCDC No. 2071259) was obtained from mixed solution of acetonitrile and water (Figure 1C, S3 and S4). Compared with the single crystal of quinazolinone-pyridine ligand (QPy-TPA) (Figure 1B), the $\pi-\pi$ interaction between BODIQPy-TPA molecules in crystal is weaker due to the hydrogen bonding between BODIQPy-TPA and water molecules, which leads to the change of molecular packing mode in the crystal. The absorption and emission spectra of BODIQPy-TPA and QPy-TPA in different solvents were then examined. The maximum absorption wavelength of BODIQPy-TPA $(\approx 488$ $\mathrm{nm})$ and QPy-TPA $(\approx 400 \mathrm{~nm})$ did not change much in the selected solvents (Figure S5 and S7). Because of the better conjugation of $\mathrm{BF}_{2}$ complex, compared with QPy-TPA, the absorption and emission wavelengths of BODIQPy-TPA red shifted (Table 1, S2 and S3). The maximum emission wavelength of BODIQPy-TPA in different solvents is above $640 \mathrm{~nm}$ except in toluene (Figure 2A, Table 1), which is located in the red/NIR region. Due to the D- $\pi$-A structure, both BODIQPy-TPA and QPy-TPA exhibited good solvochromic fluorescence properties (Figure 2A, 2B and Figure S6, S8). And BODIQPy-TPA also showed response to polarity (Figure 2F and Figure S8). The fluorescence quantum yield of BODIQPy-TPA and QPy-TPA was subsequently analyzed in different solvents (Table 1 and S2). BODIQPy-TPA showed very good emission efficiency in low polar solvent such as toluene and dichloromethane (Table 1). Although the emission efficiency of BODIQPy-TPA decreases in polar solvents such as DMSO, BODIQPy-TPA also showed good fluorescence in the DMSO/water (DMSO: $\mathrm{H}_{2} \mathrm{O}=4: 6$ ) or THF/water (THF: $\mathrm{H}_{2} \mathrm{O}=1: 9$ ) mixed solvent (Figure 2b, $\mathrm{S} 9$ and Table S4), due to the aggregation 
induced emission (AIE) characteristics of TPA group. In addition, the emission intensity of BODIQPy-TPA significantly increased in the DMSO/glycerol system (Figure 2E-2G), which further indicated the AIE property of BODIQPy-TPA due to the suppression of the rotation of TPA group. It is worth noting that BODIQPy-TPA also showed good photostability (Figure S10) and considerable two-photon fluorescence (Figure S11), which can be used for two-photon fluorescence bioimaging.
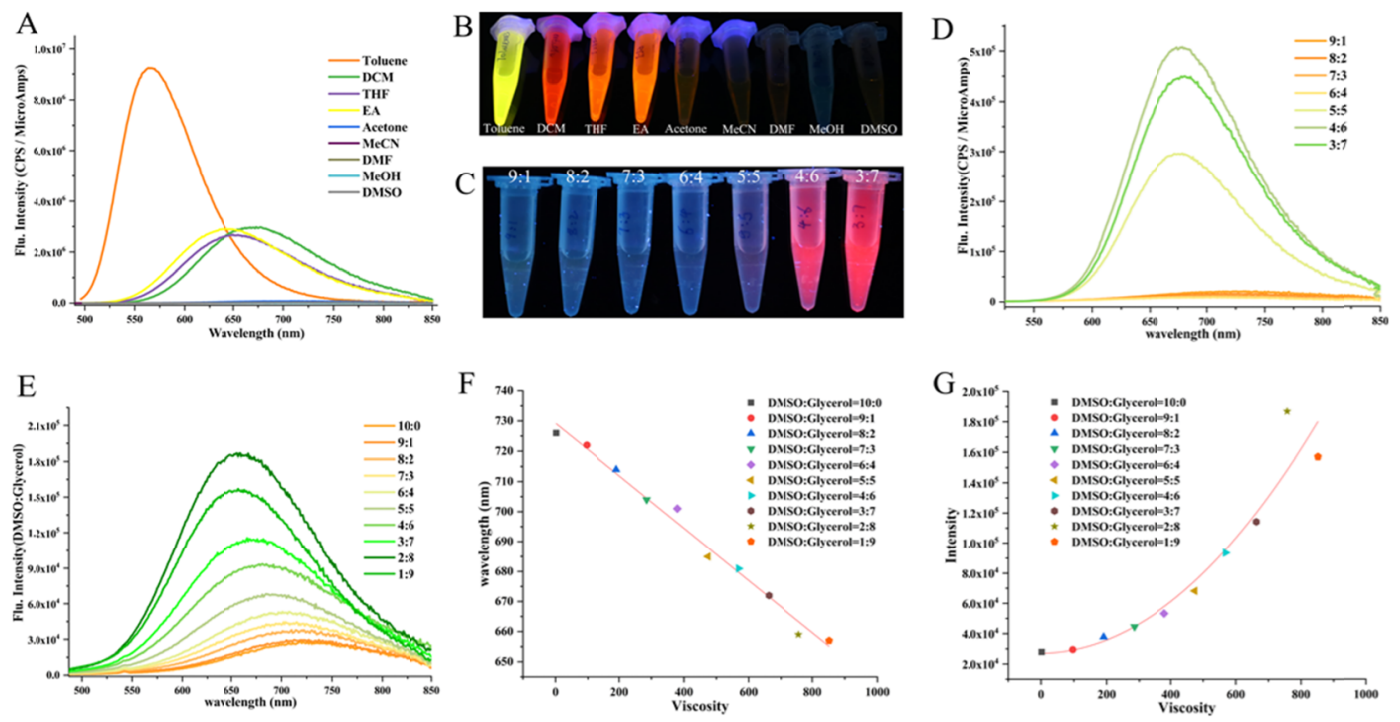

Figure 2. The luminescent properties of BODIQPy-TPA. (A) The emission spectra of BODIQPy-TPA in different organic solvents. (B) The pictures of BODIQPy-TPA in organic solutions excited at $365 \mathrm{~nm}$ using a UV lamp. (C) The pictures of BODIQPy-TPA in the DMSO/water mixed solvent. (D) The emission spectra of BODIQPy-TPA in the DMSO/water mixed solvent. (E) The emission spectra of BODIQPy-TPA in the DMSO/glycerol system. (F) The relationship of BODIQPy-TPA between maximum emission wavelength and viscosity. (G) The relationship of BODIQPy-TPA between emission intensity and viscosity.

Table 1. The optical properties of BODIQPy-TPA in solution and solid state.

\begin{tabular}{ccccccc}
\hline Solvent & $\lambda_{\text {abs }}{ }^{\max [\mathrm{a}]}(\mathrm{nm})$ & $\varepsilon\left(\mathrm{M}^{-1} \mathrm{~cm}^{-1}\right)$ & $\lambda^{\mathrm{F}}{ }_{\mathrm{ex}}{ }^{\mathrm{cc}]}(\mathrm{nm})$ & $\lambda_{\mathrm{em}}^{\mathrm{F}}(\mathrm{nm})$ & Stokes Shift $(\mathrm{nm})$ & $\Phi_{\mathrm{F}}{ }^{(\mathrm{c})}$ \\
\hline Toluene & 474 & 31500 & 476 & 566 & 92 & 0.99 \\
DCM & 486 & 33600 & 485 & 673 & 187 & 0.57 \\
THF & 467 & 38300 & 465 & 650 & 183 & 0.55 \\
EA & 462 & 43000 & 460 & 643 & 181 & 0.41 \\
DMSO & 473 & 19600 & 478 & 724 & 251 & $<0.01$ \\
DMSO:H $\mathrm{H}_{2} \mathrm{O}(4: 6)$ & 487 & 18200 & 473 & 677 & 190 & 0.10 \\
Solid & 430 & & 539 & 650 & 220 & 0.23 \\
\hline
\end{tabular}




\section{Cell imaging and ER localization of BODIQPy-TPA}

On the basis of the good emission of BODIQPy-TPA in low polar solvent and aggregation state, the fluorescence cell imaging experiments with BODIQPy-TPA were conducted in live B16 cells. As showed in Figure 3, BODIQPy-TPA exhibited good cell membrane permeability with bright green and red/NIR fluorescence in B16 cells excited by both one and two-photon (Figure 3 and Figure S12). The QPy-TPA also showed blue and green fluorescence in B16 cells. The colocalization imaging experiments were further performed using commercial dyes (ER Tracker ${ }^{\circledR}$ Blue for ER imaging and Nile Red for lipid droplets imaging). Interestingly, BODIQPy-TPA and QPy-TPA showed different subcellular localization abilities. As shown in Figure 3, BODIQPy-TPA was mainly distributed in ER with Pearson's coefficient of 0.87 , whereas QPy-TPA was mainly distributed in lipid droplets (LDs) with Pearson's coefficient of 0.86. Base on the recent literature[35], although both LDs and ER contain the lipid substances, the water content is significantly different. Based on the crystal structure analysis of BODIQPy-TPA and QPy-TPA, (Figure 1B and 1C), some water molecules was observed to form hydrogen bonds with BODIQPy-TPA, and water molecules were not found in the crystal structure of QPy-TPA, which indicated that BODIQPy-TPA showed better interaction with water than QPy-TPA. Therefore, the different hydrophilic ability of BODIQPy-TPA and QPy-TPA may be the reason for their differences in organelle localization.
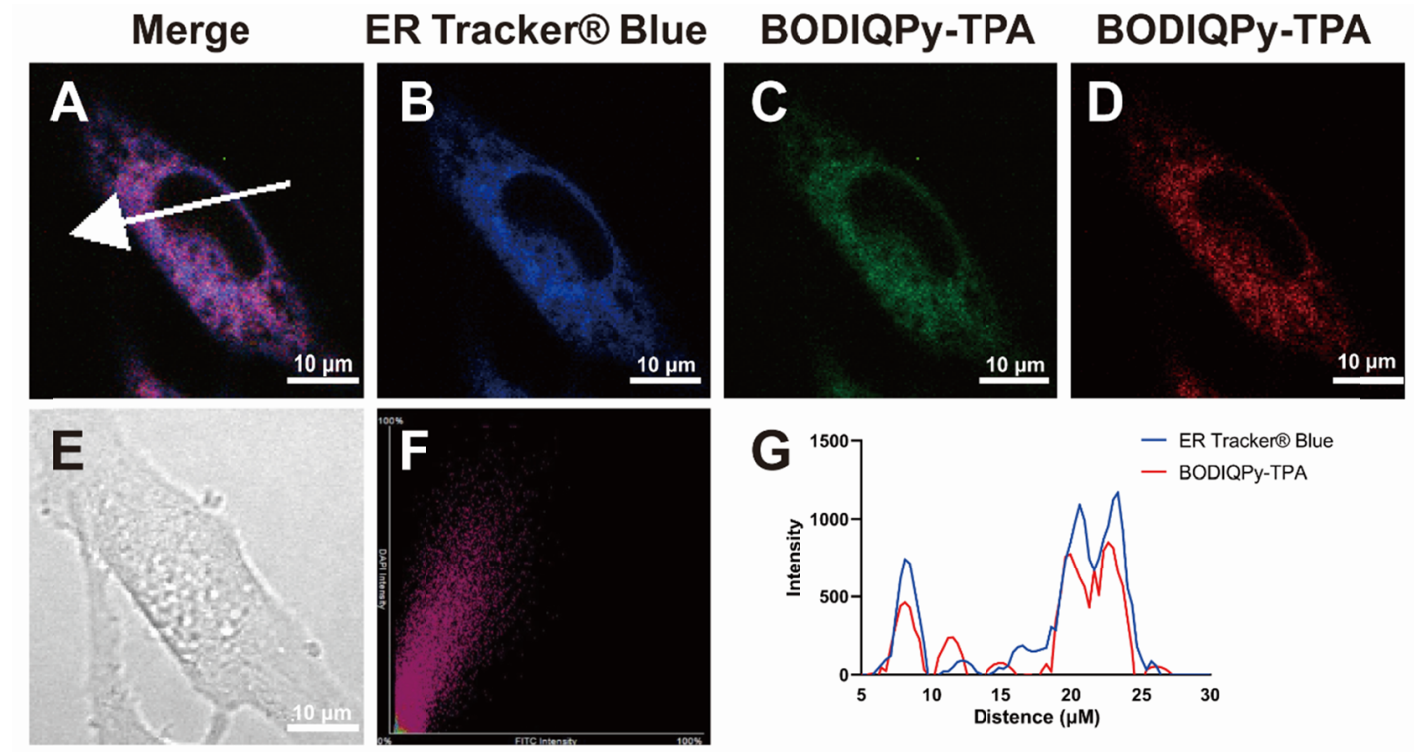

Figure 3. One-photon confocal fluorescent images of BODIQPy-TPA in Bi16 cells and the colocalization with ER traker. (A) Merged image. (B) ER Tracker®Blue (200 nM, $30 \mathrm{~min}$ ). (C) BODIQPy-TPA signal channel collected at $500-550 \mathrm{~nm}\left(\lambda_{\text {ex }}=488 \mathrm{~nm}\right.$ ), (D) BODIQPy-TPA signal channel collected at $663-738 \mathrm{~nm}\left(\lambda_{\mathrm{ex}}=488 \mathrm{~nm}\right.$ ). (E) Bright-field image. (F) Colocalization scatter plot with a Pearson's correlation coefficient of 0.87. (G) Intensity profile of the white line in image. 

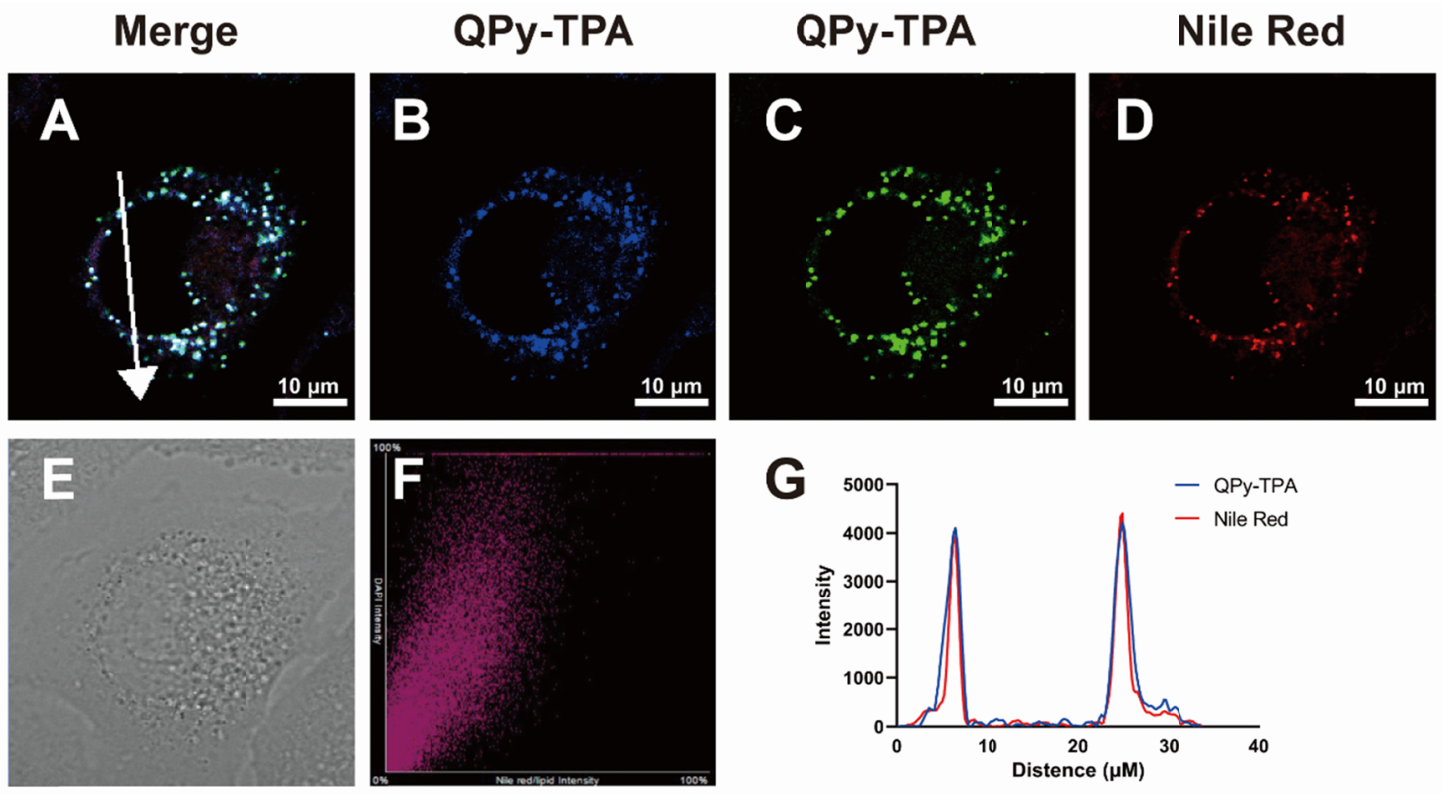

Figure 4. One-photon confocal fluorescent images of QPy-TPA in B16 cells and the colocalization with Nile Red. (A) Merge image. (B) Fluorescence image of QPy-TPA (5 $\mu$ M, Blue channel, $\lambda_{\mathrm{ex}}=405 \mathrm{~nm}$, collected $425-475 \mathrm{~nm}$ (C) Green channel, $\lambda_{\mathrm{ex}}=488 \mathrm{~nm}$, collected 500-570 nm. (D) Fluorescence image of Nile Red $\left(1 \mu \mathrm{M}, 10 \mathrm{~min}\right.$, Red channel, $\lambda_{\mathrm{ex}}=561 \mathrm{~nm}$, collected 570-620 nm) (E) Bright-field image. (F) Colocalization scatter plot with a Pearson's correlation coefficient of 0.86 . (G) Intensity profile of the white line in image.

\section{Photo-induced ferroptosis by BODIQPy-TPA}

According to the results of cell imaging, the morphology of the cells changed obviously under continuous light irradiation (Figure 5, Movie 1). The cell membrane was broken and bubbled. The nuclei of cells remained structural integrity. Interestingly, some red fluorescent circles gradually appeared in the cells at the later stage of cell death process (Figure 5 and S13). On consideration of these characteristics of cell morphological changes, the ferroptosis may occur. To determine the effect of light irradiation on the cytotoxicity of B16 cells, cell viabilities were tested with blue LED light irradiation. As shown in Figure 6, cell viability was almost unaffected by blue LED irradiation. Furthermore, the toxicity of BODIQPy-TPA (without irradiation) to B16 cells was very low $\left(\mathrm{IC}_{50}=42.11 \mu \mathrm{M}\right)$ (Figure S14A and Table S5). By comparison, BODIQPy-TPA exhibited very high cytotoxicity $\left(\mathrm{IC}_{50}=0.51 \mu \mathrm{M}\right)$ under blue LED irradiation (Figure S14B and Table S5). The photocytotoxicity index (PI), ratio of $\left(\mathrm{IC}_{50}\right)_{\text {dark }} /\left(\mathrm{IC}_{50}\right)_{\text {irrad }}$, was about 82.57, confirming good PDT effect of BODIQPy-TPA[29]. In order to further verify the process of photo-induced ferroptosis, Fer-1 as ferroptosis inhibitor was used[36,37]. As showed in Figure 6, Fer-1 prevents cells from photo-induced cell death in the presence of BODIQPy-TPA. In addition, higher concentration of Fer-1 gave better cell viability. The cell survival rate was above $80 \%$ in the presence of BODIQPy-TPA $(2.5 \mu \mathrm{M}$, with irradiation) when the concentration of Fer-1 increased to $50 \mu \mathrm{M}$. And the toxicity of BODIQPy-TPA to B16 cells was decreased $\left(\mathrm{IC}_{50}=8.87\right.$ $\mu \mathrm{M}$ ) by adding Fer-1 (Figure S14C and Table S5). More than that, western blotting results indicated that the expression of ferritin heavy polypeptide 1 (FTH1), which plays a crucial antioxidant role in the regulation of ferroptosis[38,39], was down regulated after light irradiation in the presence of BODIQPy-TPA (Figure 6B and 6C). In turn, after adding Fer-1, FTH1 expression could not be affected by light irradiation with BODIQPy-TPA. These results 
demonstrated that BODIQPy-TPA could induce ferroptosis under light irradiation. It is noteworthy that QPy-TPA also showed good photo-cytotoxicity (Figure 6D and S14B). However, Fer-1 cannot rescue cells from QPy-TPA mediated photo-induced cell death (Figure 6D and S14D), which suggested that QPy-TPA cannot cause ferroptosis under light irradiation.
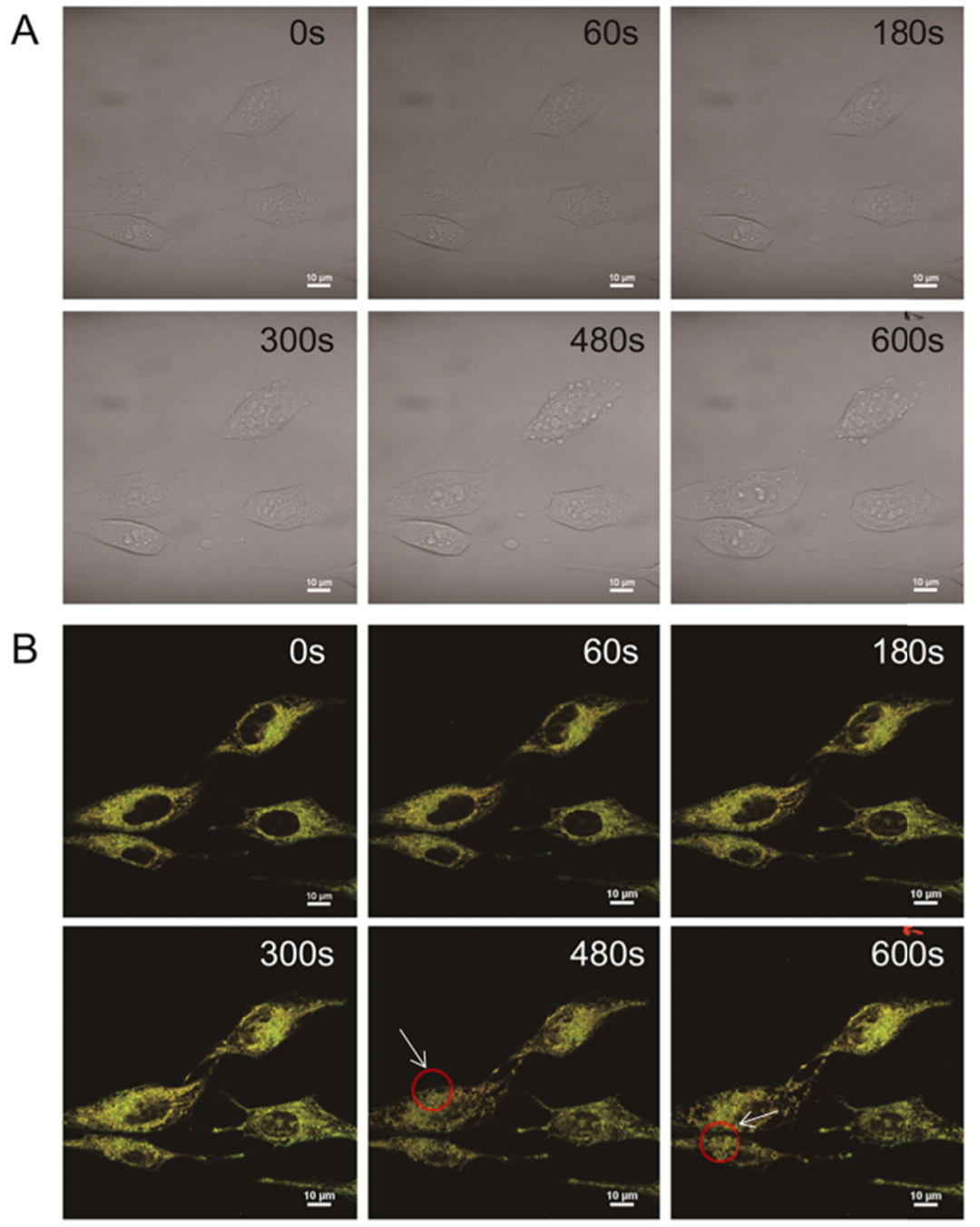

Figure 5. The morphology of the cells changed under continuous light irradiation. (A) Bright-field (B) fluorescence images change with irradiation (0-10 min) 


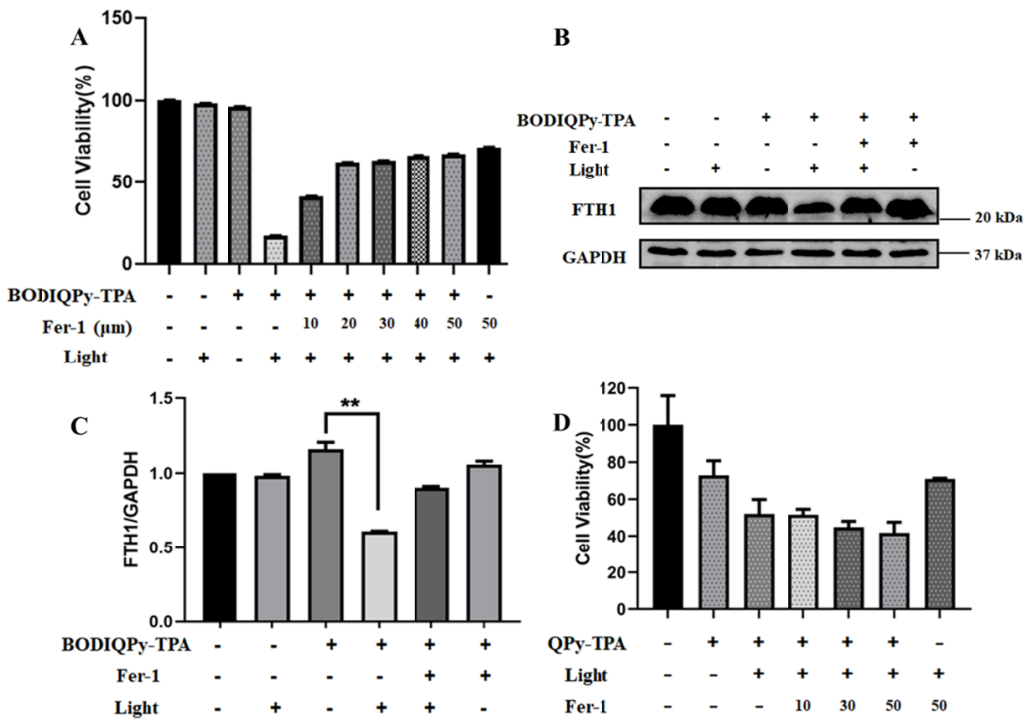

Figure 6. Photo-induced ferroptosis by BODIQPy-TPA. (A) Cell viability was measured using MTT assay with BODIQPy-TPA $(2.5 \mu \mathrm{M})$ and different doses of Fer-1. (B) Expression of FTH1 in B16 cells incubated with BODIQPy-TPA and Fer-1 after light irradiation. (C) Quantification of the immunoblot analysis in panel A. All quantitative data are presented as mean $\#$ SD from three independent experiments, and $p$ values were calculated with unpaired $t$ test; **p $<0.005$. (D) Cell viability was measured using MTT assay with QPy-TPA $(5 \mu \mathrm{M})$ and different doses of Fer-1.

\section{Photo-induced ROS production and lipid peroxidation in vitro by BODIQPy-TPA}

Given the close relationship between ferroptosis and ROS accumulation as well as the good photophysical properties of BODIQPy-TPA, we further explored the photo-triggered ROS generation of BODIQPy-TPA. Firstly, the emission spectra of BODIQPy-TPA in nitrogen and oxygen atmosphere were acquired. The fluorescence intensity of BODIQPy-TPA was enhanced in nitrogen (Figure S15), which indicated a possible energy transfer process between BODIQPy-TPA and molecular oxygen. And 1, 3-diphenylisobenzofuran (DP'BF) was used as singlet oxygen scavenger to explore the possibility of ${ }^{1} \mathrm{O}_{2}$ generation. The absorption band of DPBF decreased gradually with continuous light irradiation, further indicating the possible energy transfer process between BODIQPy-TPA and molecular oxygen (Figure S16). Subsequently, the electron paramagnetic resonance (EPR) spectroscopy was performed to detected the generation of ROS by using 2,2,6,6-tetramethylpiperidine (TEMP) and 5,5-dimethyl-1-pyrroline N-oxide (DMPO) as trapping agents. As shown in Figure 7A, the three characteristic peaks of 2,2,6,6-tetramethyl-4-piperidinol-N-oxyl (TEMPO) in EPR spectra, which are derived from oxidation of TEMP by ROS[27,40], were observed in dichloromethane solution of BODIQPy-TPA under visible light irradiation. And the signal intensity increased with the increase of visible light irradiation time (Figure S17). In the same vein, obvious signals were detected in mixed solution (DMSO/ $\mathrm{H}_{2} \mathrm{O}=6: 4$ ) of BODIQPy-TPA under visible light irradiation by using DMPO as trapping agent (Figure 7B and S18). The typical spin-trapping adduct DMPO- $\bullet O O H$ can be identified through simulation $\left(\mathrm{g}=2.0057, \alpha^{\mathrm{N}}=14.07 \mathrm{G}, \alpha^{\mathrm{H}}=10.99 \mathrm{G}\right)$, which may be attributed to peroxy radical or superoxide radical[41,42]. Accordingly, BODIQPy-TPA exhibited good photo-incudced ROS generation in organic solvent and aqueous solution probably through a combination of energy transfer and electron transfer processes. In 
consideration of the photo-toxicity of QPy-TPA, the photo-induced ROS production by QPy-TPA was also investigated through EPR spectroscopy using DMPO and TEMP as trapping agents. Similar results to BODIQPy-TPA were obtained (Figure S19), suggesting that QPy-TPA also has the ability to generate ROS.
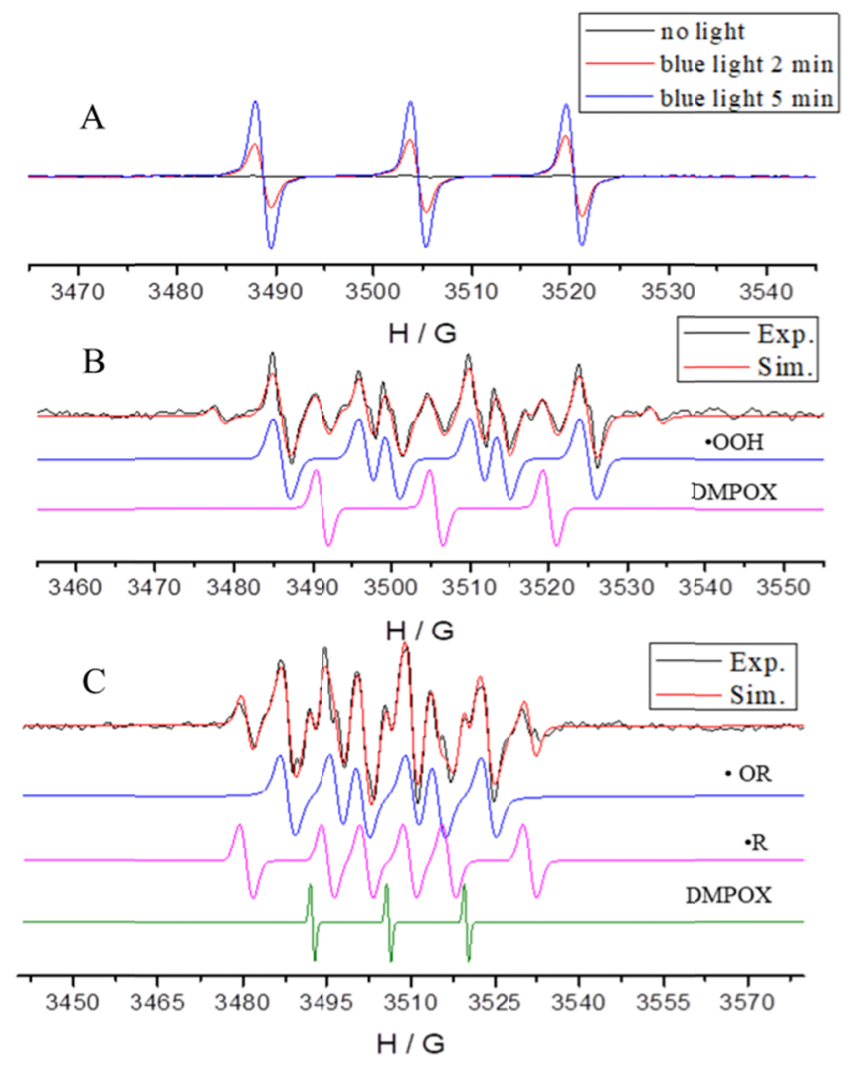

Figure 7. EPR spectra of BODIQPy-TPA under visible light irradiation (A) TEMP as trapping agent in $\mathrm{CH}_{2} \mathrm{Cl}_{2}$; (B) DMPO as trapping agent in DMSO/ $\mathrm{H}_{2} \mathrm{O}=6: 4$; (C) DMPO as trapping agent with oleic acid in $\mathrm{CH}_{2} \mathrm{Cl}_{2}$

In addition, oleic acid was chosen to explore the possible formation of lipid peroxide under light irradiation by BODIQPy-TPA. As showed in Figure 7C, two major spin trapped adducts of DMPO-•OR (hyperfine splitting constants, $\alpha^{\mathrm{N}}=13.45 \mathrm{G}, \alpha^{\mathrm{H}}=8.95 \mathrm{G}$ ) and DMPO-•R (hyperfine splitting constants, $\alpha^{\mathrm{N}}=14.60 \mathrm{G}, \alpha^{\mathrm{H}}=21.51 \mathrm{G}$ ) was well resolved after simulation[43,44]. Furthermore, the conversion of oleic acid by visible light irradiation in the presence of BODIQPy-TPA was investigated via HPLC analysis (Figure S20-S26) and ESI-HRMS. A new peak appeared at retention time of $4.59 \mathrm{~min}$ (Figure 8A and S23). And the intensity of this new peak increased gradually with the the increase of illumination time (Figure S21). This new peak was then collected and was further characterized by ESI-HRMS. As shown in Figure 8B and 8C, three major peaks with $\mathrm{m} / \mathrm{z} 281.2489,295.2280$ and 313.2384 were detected under negative ion detection, which could be attributed to oleic acid and two possible lipid peroxidation products (carbonylation products of oleic acid and peroxide of oleic acid) respectively (Figure 8C and S27). More than this, the characteristic peaks of two peroxidation products were also detected in the reaction mixture by ESI-HRMS under positive ion detection mode (Figure S27). To further investigate whether the electron transfer process is energetically favorable, Gibbs energy calculated by the Rehm-Weller equation (Eq. S1)[45]. The $\mathrm{E}^{\text {ox }}$ (Oleic acid $=-0.725 \mathrm{~V}$ ) and $\mathrm{E}^{\text {red }}$ $($ BODIQPy-TPA $=0.709 \mathrm{~V})$ were measured by cyclic voltammetry (Figure S28). $\mathrm{E}_{00}(2.20 \mathrm{eV})$ is 
the zero-zero transition energy, which can be calculated from the cross-point of the absorption and luminescence spectra at $563 \mathrm{~nm}\left(\mathrm{E}_{00}=1240 / 563 \mathrm{~nm}\right.$ in eV, Figure S29). The calculated Gibbs energy $(\Delta \mathrm{G})$ is $-3.634 \mathrm{eV}$, which suggested that the electron transfer process between BODIQPy-TPA and oleic acid is favorable. These results imply that BODIIPY-TPA could effectively promote the formation of ROS and lipid peroxidation process in vitro, which may be the main factor of photo-induced ferroptosis.

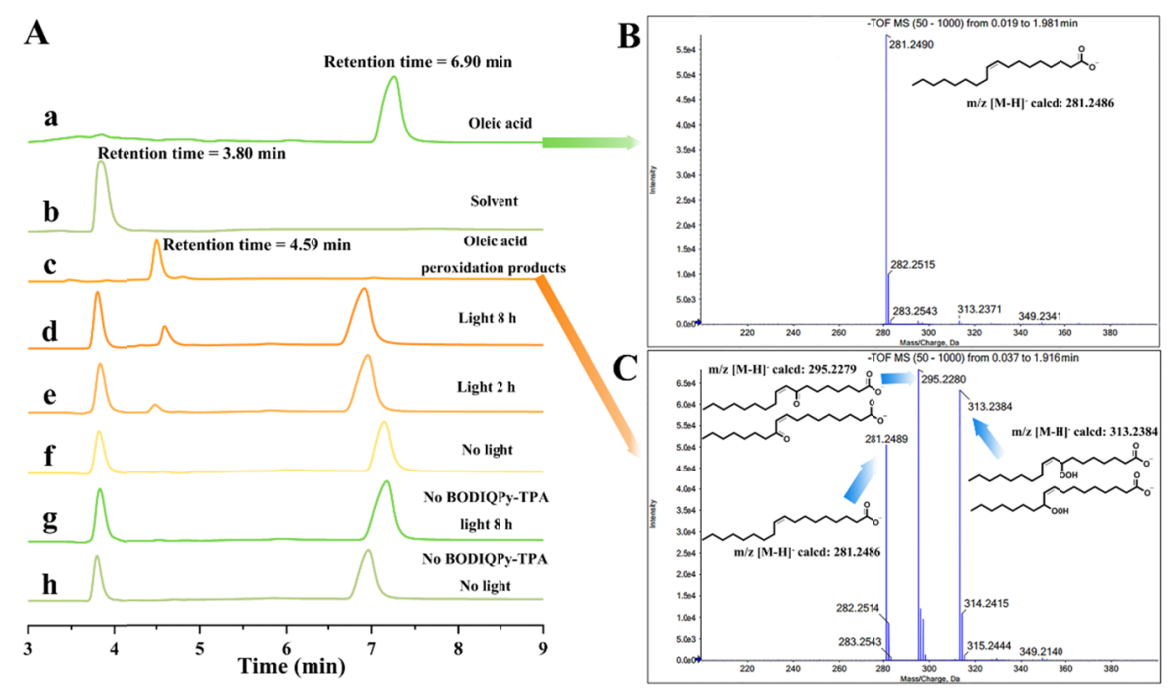

Figure 8. HPLC analysis and ESI-HRMS for the conversion of oleic acid by visible light irradiation in the presence of BODIQPy-TPA

\section{Conclusion}

In summary, vinyltriphenylamine modified quinazolinone-pyridine difluoroboron complex (BODIQPy-TPA) was developed as a photo-induced ferroptosis and NIR bioimaging bifunctional probe. The D- $\pi$-A structure of BODIQPy-TPA not only showed good red/NIR fluorescence and large Stokes shift (>90 nm) in low polar organic solvents (green fluorescence in toluene), but also showed aggregation induced fluorescence enhancement (AIEE) properties in aqueous solutions. BODIQPy-TPA exhibited bright green and red/NIR emission in live cells by one or two photon excitation. Benefiting from the good interaction between quinazolinone-pyridine difluoroboron moiety and $\mathrm{H}_{2} \mathrm{O}$ via hydrogen bond formation as well as the good lipophilic affinity of triphenylamine, BODIQPy-TPA mainly located in endoplasmic reticulum. More importantly, BODIQPy-TPA could effectively induce ROS and lipid peroxide generation under visible light irradiation, which might be the cause of ferroptosis. Therefore, BODIQPy-TPA would be a good candidate of visual probe for further investigation of the direct relationship between lipid peroxidation accumulation in ER and ferroptosis. Considering that ferroptosis could improve the efficiency of PDT for apoptosis-resistant tumor cells, we anticipate that these efforts also provide a promising strategy for the design of novel PDT agents.

\section{Accession Codes}

CCDC 2014059 and 2071259 contain the supplementary crystallographic data for this paper. These data can be obtained free of charge via www.ccdc.cam.ac.uk/ data_request/cif, or by emailing data_request@ccdc.cam.ac.uk, or by contacting The Cambridge Crystallographic Data Centre, 12 Union Road, Cambridge CB2 1EZ, UK; fax: +44 1223336033. 


\section{Supporting Information}

Supporting Information is available.

\section{Conflict of Interest}

There is no conflict of interest to report.

\section{Acknowledgements}

We are thankful for financial support from the National Natural Science Foundation of China (No. 21606116, 31871365), the Department of Education of Jiangxi Province (No. GJJ170176), and the Fundamental Research Funds for the Central Universities.

\section{References}

1. Dixon, S. J.; Lemberg, K. M.; Lamprecht, M. R.; Skouta, R.; Zaitsev, E. M.; Gleason, C. E.; Patel, D. N.; Bauer, A. J.; Cantley, A. M.; Yang, W. S.; Morrison, B., 3rd; Stockwell, B. R. Ferroptosis: An Iron-Dependent Form of Nonapoptotic Cell Death. Cell 2012, 149, 1060-1072.

2. Dixon, S. J.; Stockwell, B. R. The role of iron and reactive oxygen species in cell death. Nat. Chem. Biol. 2014, 10, 9-17.

3. Conrad M.; Pratt D. A. The chemical basis of ferroptosis. Nat. Chem. Bio. 2019, 15, 1137-1147.

4. Stockwell B. R.; Jiang X. The Chemistry and Biology of Ferroptosis. Cell Chem. Bio. 2020, $27,365-375$.

5. Wang W.; Green, M.; Choi, J.E.; Gijón M.; Kennedy P. D.; Johnson J.K.; Liao P.; Lang X.; Kryczek I.; Sell A.; Xia H.; Zhou J.; Li G.; Li J,; Li W.; Wei S.; Vatan L.; Zhang H.; Szeliga W.; Gu W.; Liu R.; Lawrence T. S.; Lamb C.; Tanno Y.; Cieslik M.; Stone E.; Georgiou G.; Chan T. A., Chinnaiyan A.; Zou W. $\mathrm{CD}^{+}$T cells regulate tumour ferroptosis during cancer immunotherapy. Nature 2019, 569, 270-274.

6. Zou Y.; Schreiber S. L. Progress in Understanding Ferroptosis and Challenges in Its Targeting for Therapeutic Benefit. Cell Chem. Bio. 2020, 27, 463-471.

7. Su Y.; Zhao B.; Zhou L.; Zhang Z.; Shen Y.; Lv H.; AlQudsy L. H. H.; Shang P. Ferroptosis, a novel pharmacological mechanism of anti-cancer drugs. Cancer Lett. 2020, 483, 127-136.

8. Shen Z.; Song J.; Yung B. C.; Zhou Z.; Wu A.; Chen X. Emerging Strategies of Cancer Therapy Based on Ferroptosis. Adv. Mater. 2018, 1704007;

9. Liang C.; Zhang X.; Yang M.; Dong X.; Recent Progress in Ferroptosis Inducers for Cancer Therapy. Adv. Mater. 2019, 1904197.

10. Yang W. S.; SriRamaratnam R.; Welsch M. E.; Shimada K.; Skouta R.; Viswanathan V. S.; Cheah J. H.; Clemons P. A.; Shamji A. F.; Clish C. B.; Brown L. M.; Girotti A. W.; Cornish V. W.; Schreiber S. L.; Stockwell B. R. Regulation of Ferroptotic Cancer Cell Death by GPX4. Cell 2014, 156, 317-331.

11. Friedmann Angeli J. P.; Schneider M.; Proneth B.; Tyurina Y. Y.; Tyurin V. A.; Hammond V. J.; Herbach N.; Aichler M.; Walch A.; Eggenhofer E.; Basavarajappa D.; Rådmark O.; Kobayashi S.; Seibt T.; Beck H.; Neff F.; Esposito I.; Wanke R.; Förster H.; Yefremova O.; Heinrichmeyer M.; Bornkamm G. W.; Geissler E. K.; Thomas S. B.; Stockwell B. R.; O’Donnell V. B.; Kagan V. E.; Schick J. A.; Conrad M. Nat. Cell Bio. 2014, 16, 1180-1191. 
12. Shimada, K., Skouta, R., Kaplan, A., Yang, W.S., Hayano, M., Dixon, S.J., Brown, L.M., Valenzuela, C.A., Wolpaw, A.J., and Stockwell, B.R. Global survey of cell death mechanisms reveals metabolic regulation of ferroptosis. Nat. Chem. Biol. 2016, 12, 497-503.

13. Bersuker K.; Hendricks J. M.; Li Z.; Magtanong L.; Ford B.; Tang P. H.; Roberts M. A.; Tong B.; Maimone T. J.; Zoncu R.; Bassik M. C.; Nomura D. K.; Dixon S. J.; Olzmann J. A. The CoQ oxidoreductase FSP1 acts parallel to GPX4 to inhibit ferroptosis. Nature 2019, 575, 688-692.

14. Ou C.; Na W.; Ge W.; Huang H.; Gao F.; Zhong L.; Zhao Y.; Dong X. Biodegradable Charge-Transfer Complexes for Glutathione Depletion Induced Ferroptosis and NIR-II Photoacoustic Imaging Guided Cancer Photothermal Therapy. Angew. Chem. Int. Ed. 2021, 60, 8157-8163.

15. Liu, T.; Liu, W.; Zhang, M.; Yu, W.; Gao, F.; Li, C.; Wang, S. B.; Feng, J.; Zhang, X. Z. Ferrous-Supply-Regeneration Nanoengineering for Cancer-Cell-Specific Ferroptosis in Combination with Imaging-Guided Photodynamic Therapy. ACS Nano 2018, 12, 12181-12192.

16. Xu T.; Ma Y.; Yuan Q.; Hu H.; Hu X.; Qian Z.; Rolle J. K.; Gu Y.; Li S. ACS Nano 2020, 14, 3414-3425.

17. Xue C.; Li M.; Liu C.; Li Y.; Fei Y.; Hu Y.; Cai K.; Zhao Y.; Luo Z. NIR-Actuated Remote Activation of Ferroptosis in Target Tumor Cells through a Photothermally Responsive Iron-Chelated Biopolymer Nanoplatform. Angew. Chem. Int. Ed. 2021, 60, 8938-8947.

18. Shah R.; Shchepinov M. S.; Pratt D. A. Resolving the Role of Lipoxygenases in the Initiation and Execution of Ferroptosis. ACS Cent. Sci. 2018, 4, 387-396.

19. Gao M.; Yi J.; Zhu J.; Minikes A. M.; Monian P.; Thompson C. B.; Jiang X. Role of Mitochondria in Ferroptosis. Mol. Cell 2019, 73, 354-363.

20. Wang H.; Liu C.; Zhao Y.; Gao G. Mitochondria regulation in ferroptosis. Eur. J. Cell Biol. 2020, $99,151058$.

21. Zou Y.; Li H.; Graham E. T.; Deik A. A.; Eaton J. K.; Wang W.; Sandoval-Gomez G.; Clish C. B.; Doench J. G.; Schreiber S. L. Cytochrome P450 oxidoreductase contributes to phospholipid peroxidation in ferroptosis. Nat. Chem. Biol. 2020, 16, 302-309.

22. Yan B.; Ai Y.; Sun Q.; Ma Y.; Cao Y.; Wang J.; Zhang Z.; Wang X. Membrane Damage during Ferroptosis Is Caused by Oxidation of Phospholipids Catalyzed by the Oxidoreductases POR and CYB5R1 Mol. Cell 2021, 81, 355-369.

23. Gaschler M. M.; Hu F.; Feng H.; Linkermann A.; Min W.; Stockwell B. R. Determination of the Subcellular Localization and Mechanism of Action of Ferrostatins in Suppressing Ferroptosis. ACS Chem. Biol. 2018, 13, 1013-1020.

24. Zhao X.; Liu J.; Fan J.; Chao H.; Peng X. Recent progress in photosensitizers for overcoming the challenges of photodynamic therapy: from molecular design to application. Chem. Soc. Rev. 2021, 50, 4185-4219.

25. Li L.; Yang Z.; Fan W.; He L.; Cui C.; Zou J.; Tang W.; Jacobson O.; Wang Z.; Niu G.; Hu S.; Chen X. In Situ Polymerized Hollow Mesoporous Organosilica Biocatalysis Nanoreactor for Enhancing ROS-Mediated Anticancer Therapy. Adv. Funct. Mater. 2020, 30, 1907716.

26. Franchi D.; Amara Z. Applications of Sensitized Semiconductors as Heterogeneous Visible-Light Photocatalysts in Organic Synthesis. ACS Sustainable Chem. Eng. 2020, 8, 15405-15429. 
27. Zhou Y.; Liu W.; Xing Z.; Guan J.; Song Z.; Peng Y. External-photocatalyst-free visible-light-mediated aerobic oxidation and 1,4-bisfunctionalization of $\mathrm{N}$-alkyl isoquinolinium salts. Org. Chem. Front. 2020, 7, 2405-2413.

28. Zhou Z.; Song J.; Nie L.; Chen X. Reactive oxygen species generating systems meeting challenges of photodynamic cancer therapy. Chem. Soc. Rev. 2016, 45, 6597-6626.

29. Yuan H.; Han Z.; Chen Y.; Qi F.; Fang H.; Guo Z.; Zhang S.; He W. Ferroptosis Photoinduced by New Cyclometalated Iridium(III) Complexes and Its Synergism with Apoptosis in Tumor Cell Inhibition. Angew. Chem. Int. Ed. 2021, 60, 8174-8181.

30. Zhuang Z.; Dai J.; Yu M.; Li J.; Shen P.; Hu R.; Lou X.; Zhao Z.; Tang B. Z. Type I photosensitizers based on phosphindole oxide for photodynamic therapy: apoptosis and autophagy induced by endoplasmic reticulum stress. Chem. Sci. 2020, 11, 3405-3417.

31. Zhou J.; Liu L.; Pan Y.; Zhu Q.; Lu Y.; Wei J.; Luo K.; Fu Y.; Zhong C.; Peng Y.; Song Z. Asymmetric Difluoroboron Quinazolinone-Pyridine Dyes with Large Stokes Shift: High Emission Efficiencies Both in Solution and in the Solid State. Chem. Eur. J. 2018, 24, 17897-17901.

32. Zhou J.; Liu L.; Zhong C.; Fu Y.; Song Z.; Peng Y. Synthesis and Luminescent Properties of 6-Methoxyquinazolinone-pyridine Difluoroboron Dyes. Chin. J. Org. Chem. 2019, 39, 1444-1449.

33. Zheng X.; Zhu W.; Ni F.; Ai H.; Gong S.; Zhou X.; Sesslerd J. L.; Yang C. Simultaneous dual-colour tracking lipid droplets and lysosomes dynamics using a fluorescent probe. Chem. Sci. 2019, 10, 2342-2348.

34. Tan P.; Zhuang W.; Li S.; Zhang J.; Xu H.; Yang L.; Liao Y.; Chen M.; Wei Q. A lipid droplet targeted fluorescent probe for high-efficiency image-guided photodynamic therapy of renal cell carcinoma. Chem. Commun. 2021, 57, 1046-1049.

35. Guo L.; Tian M.; Zhang Z.; Lu Q.; Liu Z.; Niu G.; Yu X. Simultaneous Two-Color Visualization of Lipid Droplets and Endoplasmic Reticulum and Their Interplay by Single Fluorescent Probes in Lambda Mode. J. Am. Chem. Soc. 2021, 143, 3169-3179.

36. Skouta, R.; Dixon, S. J.; Wang, J.; Dunn, D. E.; Orman, M.; Shimada, K.; Rosenberg, P. A.; Lo, D. C.; Weinberg, J. M.; Linkermann, A.; Stockwell, B. R. Ferrostatins inhibit oxidative lipid damage and cell death in diverse disease models. J. Am. Chem. Soc. 2014, 136, 4551-4556.

37. Zilka O.; Shah R.; Li B.; Friedmann Angeli J.; Griesser M.; Conrad M.; Pratt D. A. On the Mechanism of Cytoprotection by Ferrostatin-1 and Liproxstatin-1 and the Role of Lipid Peroxidation in Ferroptotic Cell Death. ACS Cent. Sci. 2017, 3, 232-243.

38. Fang Y.; Chen X.; Tan Q.; Zhou H.; Xu J.; Gu Q. Inhibiting Ferroptosis through Disrupting the NCOA4-FTH1 Interaction: A New Mechanism of Action. ACS Cent. Sci. 2021, 7 , 980-989.

39. Tian Y.; Lu J.; Hao X.; Li H.; Zhang G.; Liu X.; Li X.; Zhao C.; Kuang W.; Chen D.; Zhu M.; FTH1 inhibits ferroptosis through ferritinophagy in the 6-OHDA model of Parkinson's disease. Neurotherapeutics, 2020, 17, 1796-1812.

40. Wang X.-Z.; Meng Q.-Y.; Zhong J.-J.; Gao X.-W.; Lei T.; Zhao L.-M.; Li Z.-J.; Chen B.; Tung C.-H.; Wu L.-Z. The singlet excited state of BODIPY promoted aerobic cross-dehydrogenative-coupling reactions under visible light. Chem. Commun. 2015, 51, 11256-11259. 
41. Song Z.; Huang W.; Zhou Y.; Tian Z.-Q.; Li Z.-M.; Tao D.-J. Thermally regulated molybdate-based ionic liquids toward molecular oxygen activation for one-pot oxidative cascade catalysis. Green Chem. 2020, 22, 103-109.

42. Villamena F. A.; Liu Y.; Zweier J. L. Superoxide Radical Anion Adduct of 5,5-Dimethyl-1-pyrroline N-Oxide. 4. Conformational Effects on the EPR Hyperfine Splitting Constants. J. Phys. Chem. A 2008, 112, 12607-12615.

43. Zhang J.; Liu D.; Liu S.; Ge Y.; Lan Y.; Chen Y. Visible-Light-Induced Alkoxyl Radicals Enable a-C(sp3)-H Bond Allylation. iScience 2020, 23, 100755.

44. Li F.; Xiao L.; Li Y.; Chen C.; Liu L. The interception of a copper-based carbene radical with an a-carbonyl diazomethane radical: $\mathrm{C} 1 / \mathrm{C} 1 \mathrm{~N} 2$ copolymerization. Chem. Commun. 2015, 51, 11964-11967.

45. Le H.-N.; Brazard J.; Barnoin G.; Vincent S.; Michel B. Y.; Leonard J.; Burger A. Control of Intermolecular Photoinduced Electron Transfer in Deoxyadenosine-Based Fluorescent Probes. Chem. Eur. J. 2021, 27, 1364-1373. 